\title{
Development of Ultrahigh Resolution Objective Lens Enabling High Analytical Sensitivity
}

Yu Jimbo, Ichiro Ohnishi, Hiroki Hashiguchi, Yorinobu Iwasawa, Shigeyuki Morishita, kouji miyatake, Masaki Mukai and Hidetaka Sawada

JEOL Ltd., Akishima, Tokyo, Japan

Spatial resolution of transmission electron microscope (TEM) with spherical aberration corrector has been improved to be $40 \mathrm{pm}$ [1], and it enables ordinary observation of materials samples at atomic resolution. Recently, such atomic resolution has become required not only for high resolution imaging at high accelerbut observations with a variety of methods and conditions. Among these various observations, the combination of scanning transmission electron microscopy (STEM) and energy dispersive X-ray spectroscopy (EDS) is typically popular and requested from huge number of researchers, because it gives fundamental properties of materials. The EDS sensitivity improves, when the EDS detector set at closed position to the specimen and/or the detection area of the EDS detector is large. However, the space near the specimen is limited by the objective pole-piece. Therefore, we tried to optimize the position of the detector by redesigning the objective lens polepiece of ultrahigh resolution to achieve the high resolution and EDS sensitivity simultaneously. The shape of the objective pole-piece for high resolution TEMs is generally thick to avoid the magnetic saturation. And the gap between pole-pieces is narrow to make sharp magnetic field distribution, which results in the reduction of the chromatic aberration coefficient.

We used the previously developed highly sensitive EDS system for JEM-ARM300F [2], which has large sensor area of $158 \mathrm{~mm}^{2}$, for the new objective lens polepiece design. Finally, we have developed new ultrahigh resolution pole-piece (called FHP2) optimized to the $158 \mathrm{~mm}^{2}$ EDS detector. The FHP2 can be installed to a newly designed $300 \mathrm{kV}$ aberration corrected TEM (JEOL JEM-ARM300F2). Two EDS detectors can be installed to the FHP2. The total detection solid angle is $1.4 \mathrm{sr}$ (Each detector has $0.7 \mathrm{sr}$.), and the take-off angle for both detectors is same to be $26 \mathrm{deg}$, which enables a X-ray detection at high position, that is, almost no obstacles between the sample and the detector.

Figure 1 shows STEM images and EDS elemental map of GaN [211] obtained at $300 \mathrm{kV}$ by aberration corrected $300 \mathrm{kV}$ microscope (JEM-ARM300F2 with FHP2) with the developed EDS system. Figure 1(a) and (c) show an annular bright field (ABF)-STEM and high angle annular dark field (HAADF)-STEM images. And Fig. 1(b) shows an elemental map by the highly sensitive EDS system. The convergent angles of electron probe for the three methods were $32 \mathrm{mrad}$ in semi angle. The probe current was $35 \mathrm{pA}$ for the EDS elemental map. The Ga-Ga dumbbells of $63 \mathrm{pm}$ is completely resolved in the HAADF-STEM image as shown in Fig. 1(c), and resolved Ga-Ga dumbbells can be seen in the EDS elemental map. This proves success of sub-angstrom resolution in EDS elemental map.

Additionally, spatial resolution in STEM at $60 \mathrm{kV}$ is fairly good as 75 pm with FHP2 owing to its low chromatic aberration coefficient. Therefore, the signal to noise ratio for HAADF-STEM image is high even at low acceleration voltages. Figure 2 shows a time sequential series of HAADF-STEM images for a mono-layer graphene sample obtained at $80 \mathrm{kV}$. The convergent angle of probe was $32 \mathrm{mrad}$ in semi angle. The positions of individual carbon atoms in a series of images could be clearly seen, and a double carbon chain was seen in the last image (the $4^{\text {th }}$ image). 


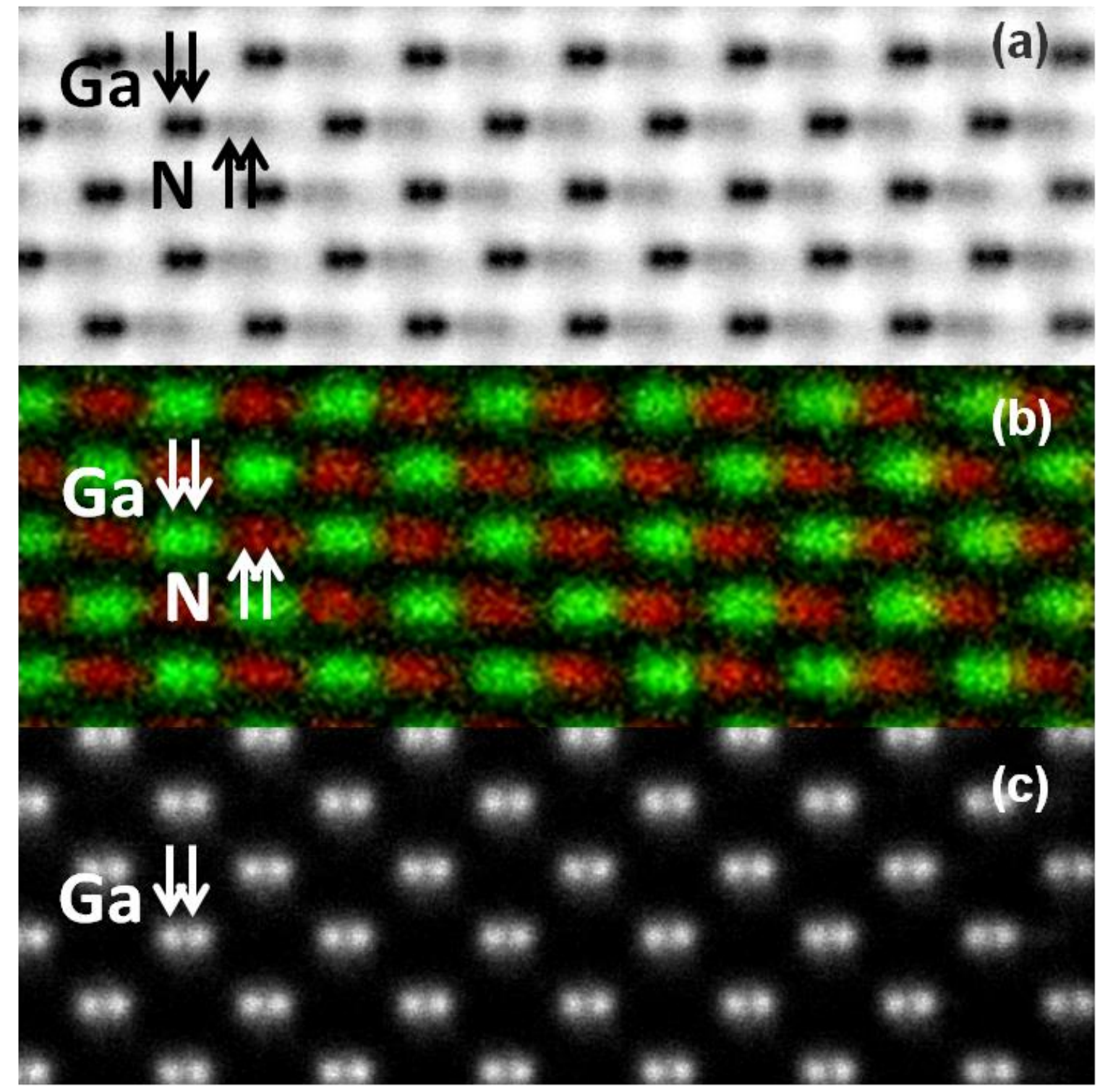

Figure 1. GaN [211] images of (a) ABF-STEM, (b) STEM-EDS elemental map and (c) HAADF-STEM obtained at $300 \mathrm{kV}$. The Ga-Ga dumbbells having $63 \mathrm{pm}$ spacings are completely resolved in the HAADFSTEM image. And the resolved dumbbells can be seen on the STEM-EDS elemental map. 

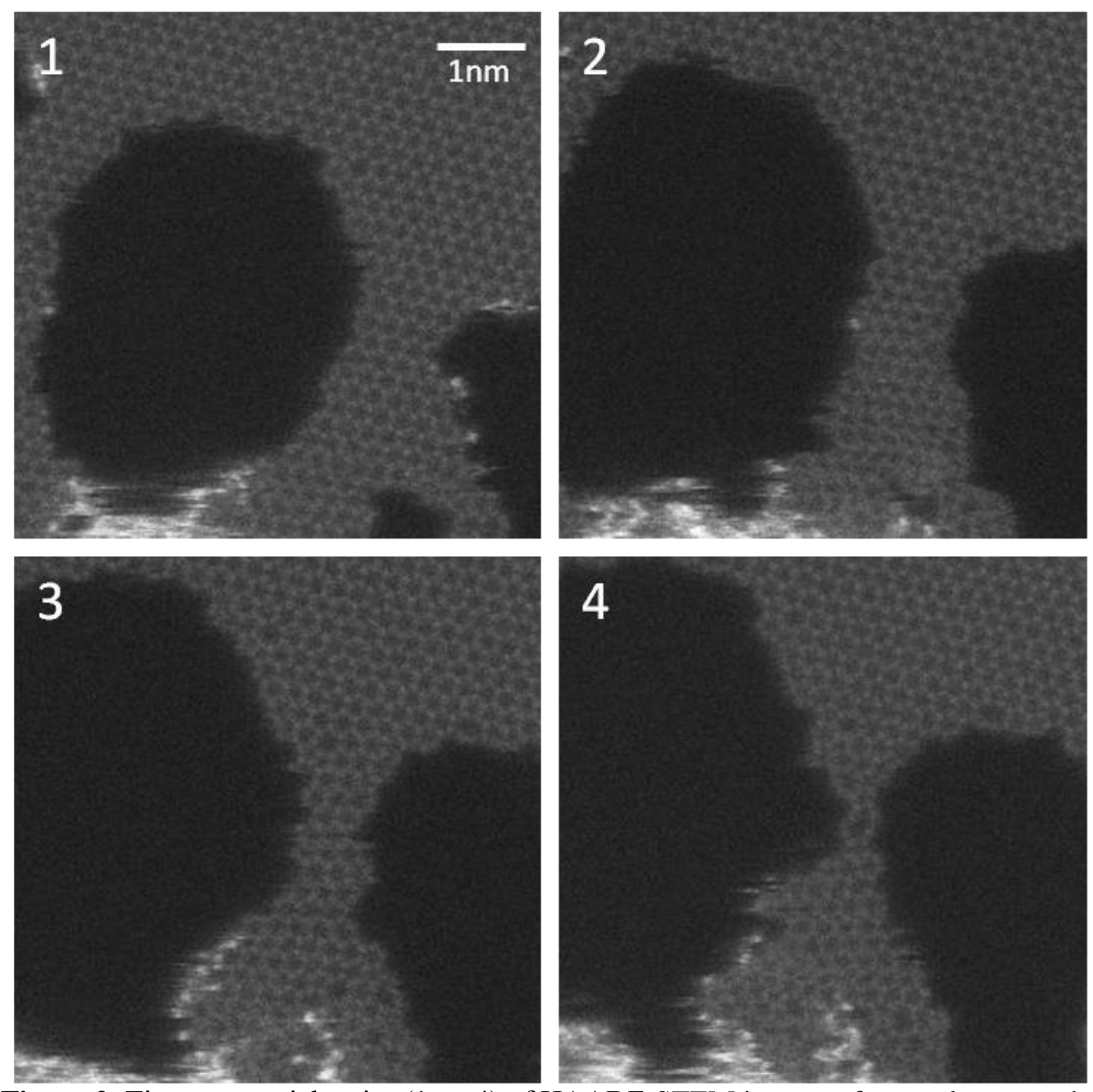

Figure 2. Time sequential series $(1 \rightarrow 4)$ of HAADF-STEM images of mono-layer graphene obtained at $80 \mathrm{kV}$. The dwell time and number of pixels were $19 \mu \mathrm{s}$ and $1024 \mathrm{x} 1024$. The positions of carbon atoms are identified clearly, owing to sub-angstrom probe formed by the FHP2 pole-piece.

\section{References}

[1] S Morishita et al, Microscopy, 67 (2018) p. 46.

[2] E.Okunishi et al., JEOL News 50, (2015)p42. 